\title{
A subject-specific software solution for the modeling and the visualization of muscles deformations
}

\author{
Xavier Maurice • Anders Sandholm • Nicolas Pronost • \\ Ronan Boulic · Daniel Thalmann
}

Published online: 3 March 2009

(C) Springer-Verlag 2009

\begin{abstract}
Today, to create and to simulate a virtual anatomical version of a subject is useful in the decision process of surgical treatments. The muscular activity is one of the factors which can contribute to abnormal movements such as in spasticity or static contracture. In this paper, we propose a numerical solution, based on the Finite Element (FE) method, able to estimate muscles deformations during contraction. Organized around a finite element solver and a volumetric environment, this solution is made of all the modeling and simulation processes from the discretization of the studied domain to the visualization of the results. The choices of materials and properties of the FE model are also presented such as the hyperelasticity, the contention model based on inter-meshes neighboring nodes pairing, and the estimation of nodal forces based on the subject-specific muscular forces and action lines.
\end{abstract}

Keywords Muscle deformation · Finite element modeling · Anatomical human visualization

\section{Introduction}

These past years, a large evolution of the human modeling techniques have emerged thanks to: the development of more elaborate mathematical models, the progress of the resolution in medical imagery or the decrease of

X. Maurice · A. Sandholm · N. Pronost $(\bowtie) \cdot$ R. Boulic .

D. Thalmann

École Polytechnique Fédérale de Lausanne - VRLab, Lausanne,

Switzerland

e-mail: nicolas.pronost@epfl.ch the simulation time on larger and more accurate anatomical models. Computational models that characterize the three-dimensional surface geometry of bones, kinematics of joints, and force-generating capacity of muscles have showed powerful methods for investigating the muscle function. Nevertheless, if the causes of some body behaviors are well identified, the prediction of the movement of most of the anatomical structures with a controlled accuracy still reveals an important issue. And being able to provide information from this kind of prediction could have a major impact on diagnostic methods in many fields relating to human modeling.

We believe that computer software used for modeling and simulating the deformation of a muscle can help provide scientific data for treating movement disorders. Predicting the deformation of a muscle allows to evaluate the internal displacements inside the muscle, that is difficult to measure experimentally. In this paper, we propose a numerical solution to model the muscular deformations during contraction.

Because of their organization, to model muscles, from the sets of fibers to the series of sarcomeres, is challenging. The first approaches tried to approximate the external shape of the muscles thanks to geometrical primitives, such as ellipsoids [22], metaballs [26] or Free From Deformation objects [24]. Their main interest lies on a fast resolution of their motions allowing real-time applications. In such models, the muscles move along a predefined trajectory far away from the real complexity of a musculoskeletal system.

The complexity of the problem requires implementation of sophisticated numerical methods lying on physics properties. Computational modeling work has included anatomical or geometrical observations [7, 11] and analytical mathematical modeling [4, 14], as well as methods working without the surface information (Mesh Free method [5]). Re- 
cently, advanced finite element modeling approaches have allowed for improvements in the predictive power of tissue deformation [12, 15], joint kinema tics [21] or the simulation of muscle fatigue [25].

Significant effort has also been placed into accelerating FEM calculations including recent approaches that precompute and cache various quantities [20], modal analysis [13], and approximations to local rotations [19]. The FE method is ideally suited for solving volumetric deformation over time. We propose to estimate internal deformation using a software pipeline that combines FE modeling and computing methods with subject-specific data. The main contribution of the paper is to present an almost automatic solution to the simulation of subject-specific deformations of muscle tissues from the initial 3D boundary geometries to the final mesh displacements.

\section{Outline of the method}

In this paper, we present the deformation software solution of a muscle during contraction. For this purpose, we use the two following subject-specific data (see Fig. 1):

- The 3D models of muscular systems reconstructed from segmented MRI, including bones, tendons, muscles and the subject-adapted action lines computed from [10];

- The estimation of the muscular forces during contraction calculated from motion capture on the same subject. These forces are computed by a process involving a subject-specific musculoskeletal modeling, inverse kinematics, inverse dynamics and residual reduction [23].

The system then provides a three-dimensional visualization of the deformation of the reconstructed muscles. The FE musculoskeletal model (see Sect. 3) is defined using the more appropriate properties. The computational method is described in Sect. 4.

\section{Finite Element musculoskeletal model}

Because of its flexibility and its physics-based approach, we model the muscles using a Finite Element (FE) method $[6,16]$. In FE terms, the analysis is anisotropic, nonlinear, applied on large deformations, quasi-static and quasiincompressible:

- Anisotropic. Indeed, the system is directionally dependent. For example, the passive physical properties of the contractive part of the muscles and the tendons may be different. The type of pennation that involves the fiber directions and thus the force moment can also be different for the same muscle.

- Nonlinear. The behavior of a muscle is clearly nonlinear, and so the majority of the current models tends to describe it by hyperelastic materials, for example by MooneyRivlin models. This property corresponds to the nonlinear change of the strain of the structure according to the applied stress.

- Large deformations. The large deformations categorize the problems in which elongations take a significant proportion related to the study area, which is true with muscles: their deformations during the contraction are often visible to the naked eye.

- Quasi-static. In a static analysis, the same force is applied to the same place in the study. In a dynamic analysis, the parameters of the study may vary over time. Here, we involve a quasi-static analysis. Hence, the inertial forces are not considered, but the load increases over the time from zero to the maximal contraction force.

- Quasi-incompressible. We consider that the volume of a muscle does not vary during contraction (the muscle still contains as much matter), which may be physiologically false, as the blood pressure can increase. We define the quasi-incompressibility property of the model by a Poisson summation formula with a factor very close to 0.5 .

Most of the current muscular models are derived from Hill's three components model [27]. The model is composed
Fig. 1 Segmented MRI are used to create the surface models of the muscles, bones and tendons. Muscular properties (action lines, materials, constraints, etc.) are then added to the models in order to produce a FE musculoskeletal model. The muscular forces are estimated from captured motions. The displacements inside the muscles are then computed and finally displayed

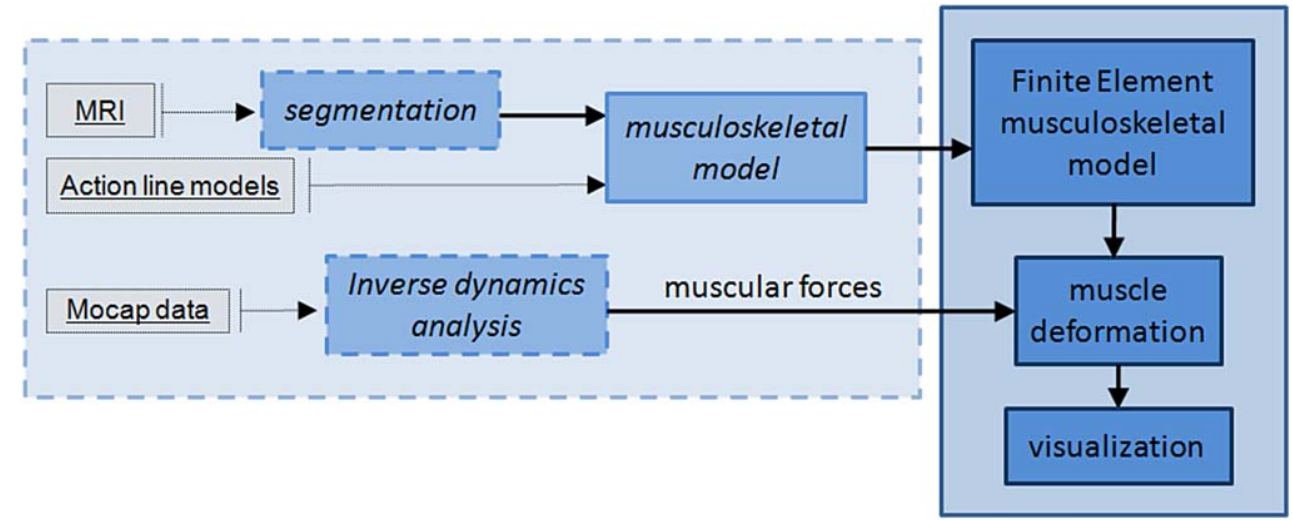


Fig. 2 Overview of the FE musculoskeletal simulation pipeline
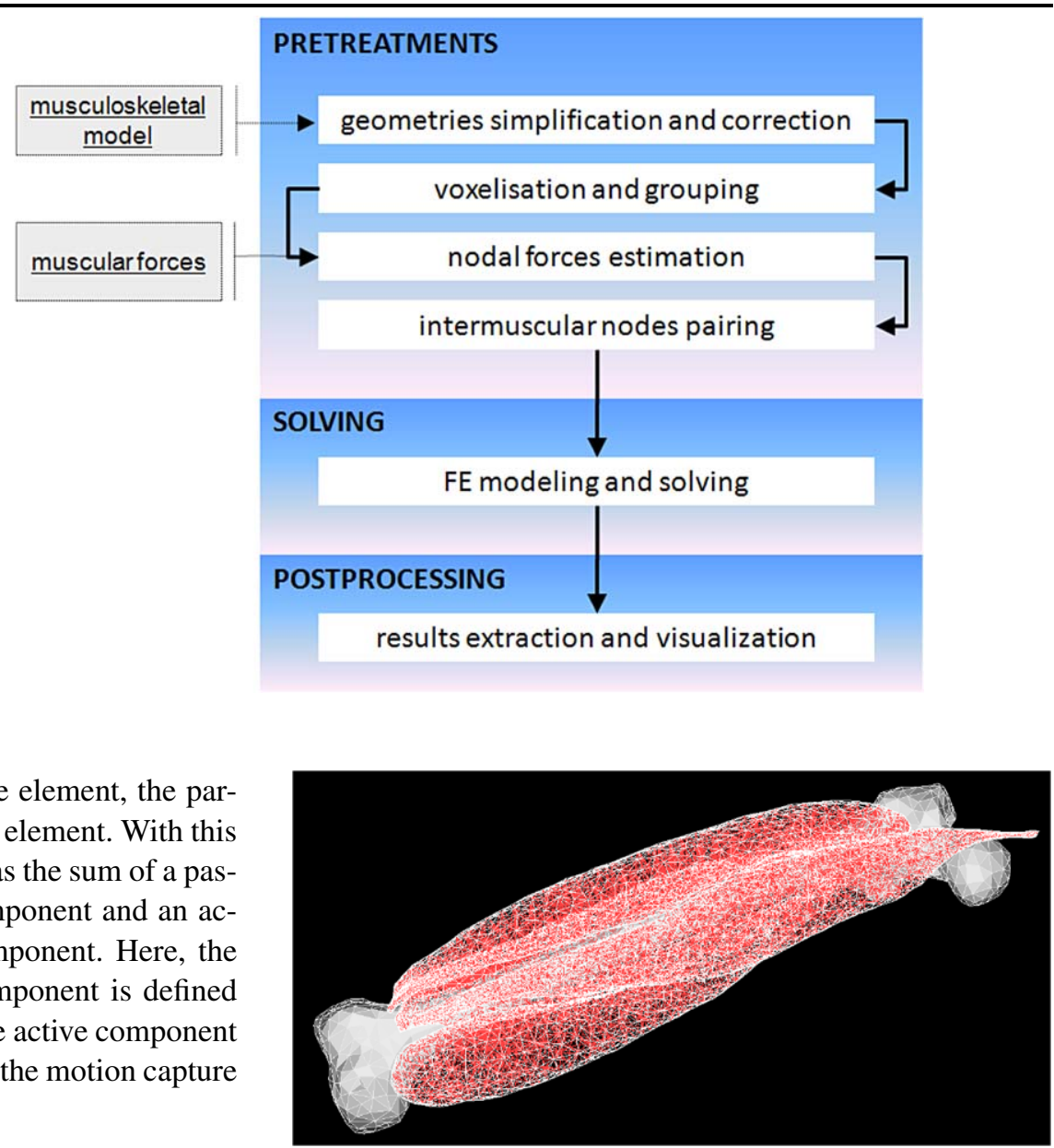

Fig. 3 Result of the voxelization of the left quadriceps femoris muscles and femur

FE system much more rapidly and to avoid cross surfaces problems in thin areas. We so focus on quality aspects, quantitative accuracies are not expected. We used an algorithm allowing the conservation of the geometry and the acute angles, developed within the Maya software. This step allows us to work on a more interactive model for the solving as well as for the visualization. The models have also been corrected and smoothed in order to avoid inter-penetration between the different structures: bones, muscles and tendons. Second, the surface models are converted into volumetric models grouping the voxels of a same structure (see Fig. 3). This process is performed within the Salome open source platform [2].

Third, using the muscular forces and the action lines (see [23]), we need to determine the contractile force direction and magnitude on each node of the muscles. These forces are called nodal forces in this work. For each node $v_{M}$ of a muscle $M$ (excluding the origin and insertion tendons areas), the direction of the nodal force $N F D_{v_{M}}$ is calculated according to the directions of the $n$ action lines $A L_{M i}, i \in[1, \ldots, n]$ and to the minimal distances between later for clinical accuracy, they were used here to solve the 

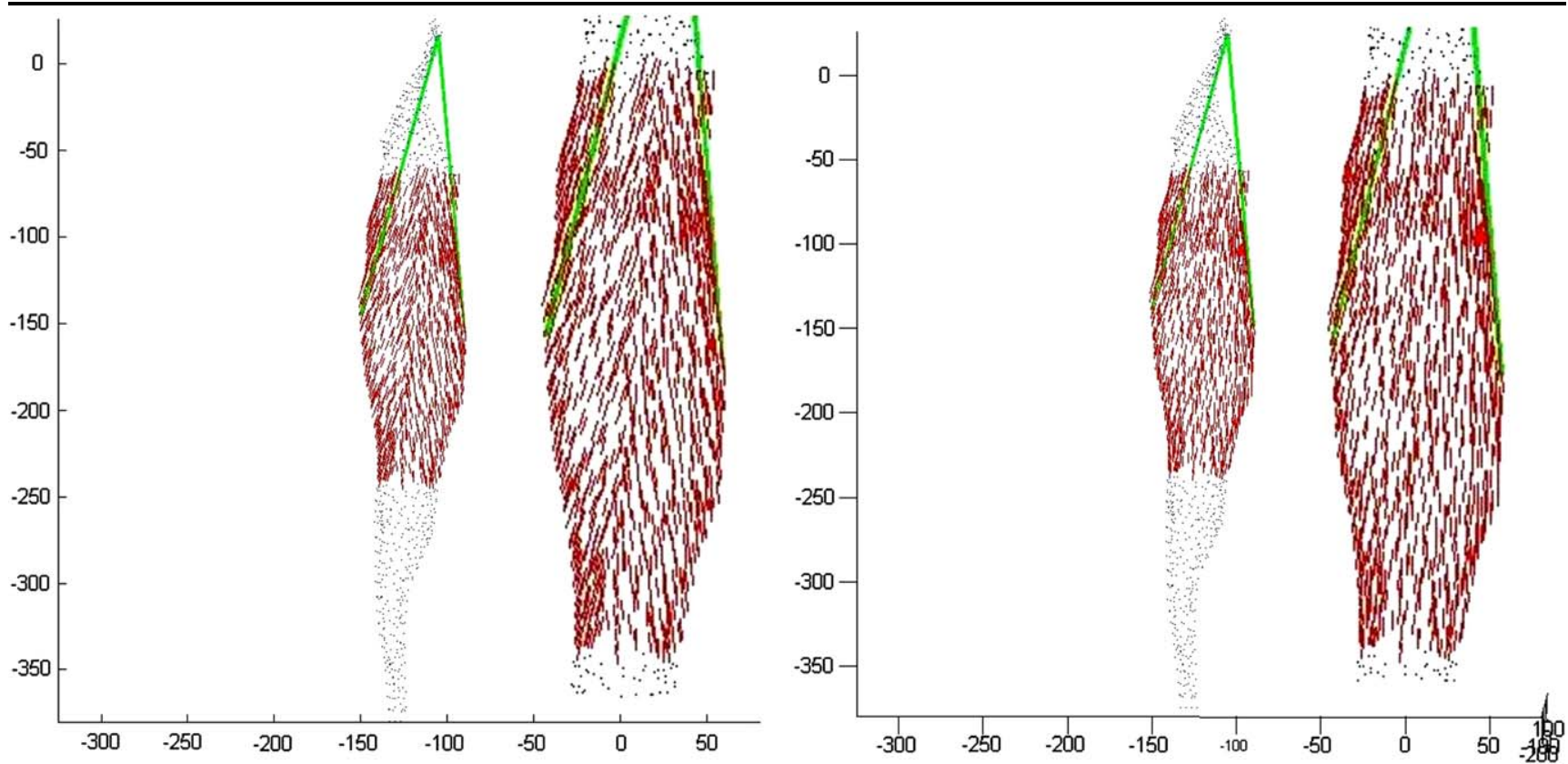

Fig. 4 Nodal forces (in red) on the left quadriceps muscle based on two action lines (in green). Left: only using the direction of the nearest line. Right: using the distance-based interpolation

$v_{M}$ and each action lines. We propose two computational methods (see Fig. 4):

1. Based only on the direction of the nearest action line of the muscle. Using a discretization of the action lines, we compute the distances between the nodes and their nearest points on the lines. The final directions are directly defined by the direction of the line corresponding to the smaller distance.

2. Based on the mix of the directions of all the action lines of the muscle. In that case, we propose a weighted sum of the direction of all the lines in the muscles. The weights $w_{v_{M}}(i)$ are linearly dependent on the minimal distances between the node and the lines:

$$
N F D_{v_{M}}=\left\|\sum_{i=1}^{n} w_{v_{M}}(i) \times A L_{M i}\right\| \text {. }
$$

Depending on the muscle we want to model, these two methods can be used to represent different types of pennation.

At each time $t$ during the contraction, the magnitude $N F M_{v_{M}}(t)$ of a nodal force is defined as the division of the magnitude of the muscular force $F_{M}(t)$ by the number of contributing elements $N_{v}$, so corresponding to an homogeneous distribution of the force in the fibers:

$N F M_{v_{M}}(t)=\frac{1}{N_{v}} \times\left\|F_{M}(t)\right\|$.

$N_{v}$ corresponds to the number of nodes contributing to the corresponding muscle. These groups of nodes are defined from the subject-specific geometry of the muscles. An interactive and graphical interface can be used by an expert in order to select these nodes.

The last step before solving the FE simulation is to define the constraints of the mechanical system. In our model, the tendons are attached to the bones and the degrees of freedom of the system only come from the volumetric positions of the nodes. Moreover, we propose an integration of the interferences which occur between neighboring muscles, modeling the contention phenomenon. Indeed, the human body does not contain air, except inside the lungs. The structures (organs, bones, muscles, etc.) are linked together, so for example, the contraction of a muscle does not create space. We propose to model this property by adding distance constraints on nodes. Pairs of nodes are defined near to the surfaces of the muscles, and the solver try to respect the initial distance between the nodes. Our software solution provides a semi-automatic process to define these pairs (see Fig. 5), based on neighboring conditions (preventing to pair nodes inside a same muscle) and thresholds (maximal distances between nodes of the different structures). In the future, image processing analysis of the subject-specific MRI images may allow the validation of this contention model. The FE solver then uses these distance relationships as constraints on the degrees of freedom of the involved nodes.

The last constraints to create are the insertions and origins areas of the tendons. An interactive and graphical interface is then used to select parts of the structures corresponding these areas. Hence, the constraints are automati- 


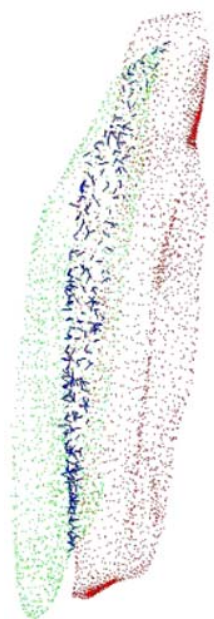

(1)

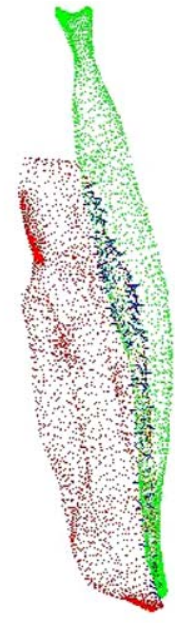

(2)

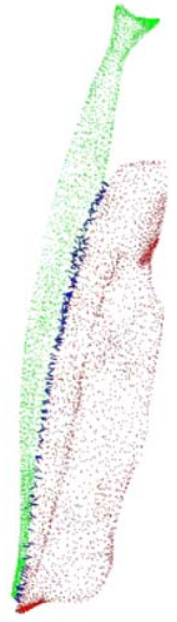

(3)
Fig. 5 Visualization of constrained pairs of nodes (blue) between (1) Vastus Medialis (green) and Vastus Intermedius (red), (2) Rectus Femoris (green) and Vastus Intermedius (red), (3) Rectus Femoris (green) and Vastus Lateralis (red)

cally placed between the bones and the tendons and between the tendons and the muscles.

\subsection{Solving}

To solve the biomechanical constraints at each time of the contraction, we use the Code-Aster platform [1]. This platform offers a full range of multiphysical analysis and modeling methods on FE models under constraints, and uses the Newton-Raphson resolution method. We describe our system by commands which are interpreted by the platform, including the materials of the volumetric data, the constraints on the degrees of freedom and the input nodal forces.

The passive component of the muscle was modeled by a hyperelastic 2-parameters Mooney-Rivlin model [17]. The parameters of this model are set up from the Signorini-type hyperelastic characteristics [9], where the Green-Lagrange deformations $\Psi$ are defined as follows:

$$
\begin{aligned}
\Psi= & C 01\left(I_{1}-3\right)+C 10\left(I_{2}-3\right)+C 20\left(I_{1}-3\right)^{2} \\
& +\frac{1}{2} K(J-1)
\end{aligned}
$$

$-I_{1}=I_{c} J^{-\frac{2}{3}}, I_{2}=I I_{c} J^{-\frac{4}{3}}, J=I I I_{c}^{\frac{1}{2}}$ and $\left(I_{c} I I_{c} I I I_{c}\right)$ are the 3 invariants of the right Cauchy-Green deformation tensor.

- C01, C10 and C20 are the three coefficients, in MPa, of the polynomial expression of the hyperelastic material. If $C 10=C 20=0$, we obtain a neo-Hookean material, but if only $C 20=0$, we obtain a Mooney-Rivlin material. So in our study, $C 20=0$, while $C 01$ and $C 10$ define the muscle properties from [8] as follows: $C 01=0.06 \mathrm{MPa}$, $C 10=0.12 \mathrm{MPa}$.
- $K$ express the compressibility of the material and is given by:

$$
K=\frac{6(C 01+C 10)}{3(1-2 v)}
$$

where $v=0.4999$ is the Poisson's ratio from [8].

The materials of the tendons are defined by a linear elasticity with a Young's modulus of $1200 \mathrm{MPa}$ and a Poisson's ratio of 0.4 [18]. The materials of the bones are defined by quasi-incompressible elasticity with a Young's modulus of $17000 \mathrm{MPa}$ and a Poisson's ratio of 0.3 [18]. These values were determined by curve fitting using mean square errors between the stress-strain function of the models and those obtained empirically from real muscles.

Working on the 12173 tetrahedrons of the quadriceps femoris model (4 muscles and 1 bone), a common laptop takes about $16 \mathrm{~s}$ to solve one frame of the simulation (around 5 iterations with an average residual error of 3.9e-11).

\subsection{Postprocessing and experimental results}

We finally extract and visualize the results of the contraction simulation. We first propose to visualize the surface displacements of the biomechanical system (see Fig. 6). We observe that the femur is static, due to its quasi-solid material as well as the insertion areas. The experiments show also that the tendons move less than the muscles, because of their higher stiffness. For the muscle, the central part is the more mobile than the extremities. These results, from a macroscopic point of view, seem to be valid but they still need to be accurately validate according to literature and clinical observations.

Figure 7 shows internal movements inside the structures of the quadriceps femoris. It represents 10 slices, perpendicular to the main axis of the femur, at 5 regular time-frames of the contraction. We can then study the nodal displacement behaviors inside of the muscles. The results show that the external muscles (Rectus Femoris, Vastus Lateralis) move more than the internal ones (Vastus Medialis, Vastus Intermedius), which can be usually observed by experimental measurements. Nevertheless, we observed also some irregularities inside a single muscle. They come from the irregularity of the sizes of the tetrahedrons from the voxelization, meaning that the discretization of the muscles is not homogeneous and so that the nodes cannot directly represent the sarcomeres of the fibers. To solve this problem, we plan to parameterize the nodal force estimation by the density of the neighboring nodes.

\section{Conclusion}

In this paper, we proposed a software solution to model and to simulate the deformation of a muscle during contraction. 
Fig. 6 Deformations of the volumetric models of the quadriceps femoris system during contraction. The distance from the original position of each node is represented from blue to red. Up: front view; Down: back view
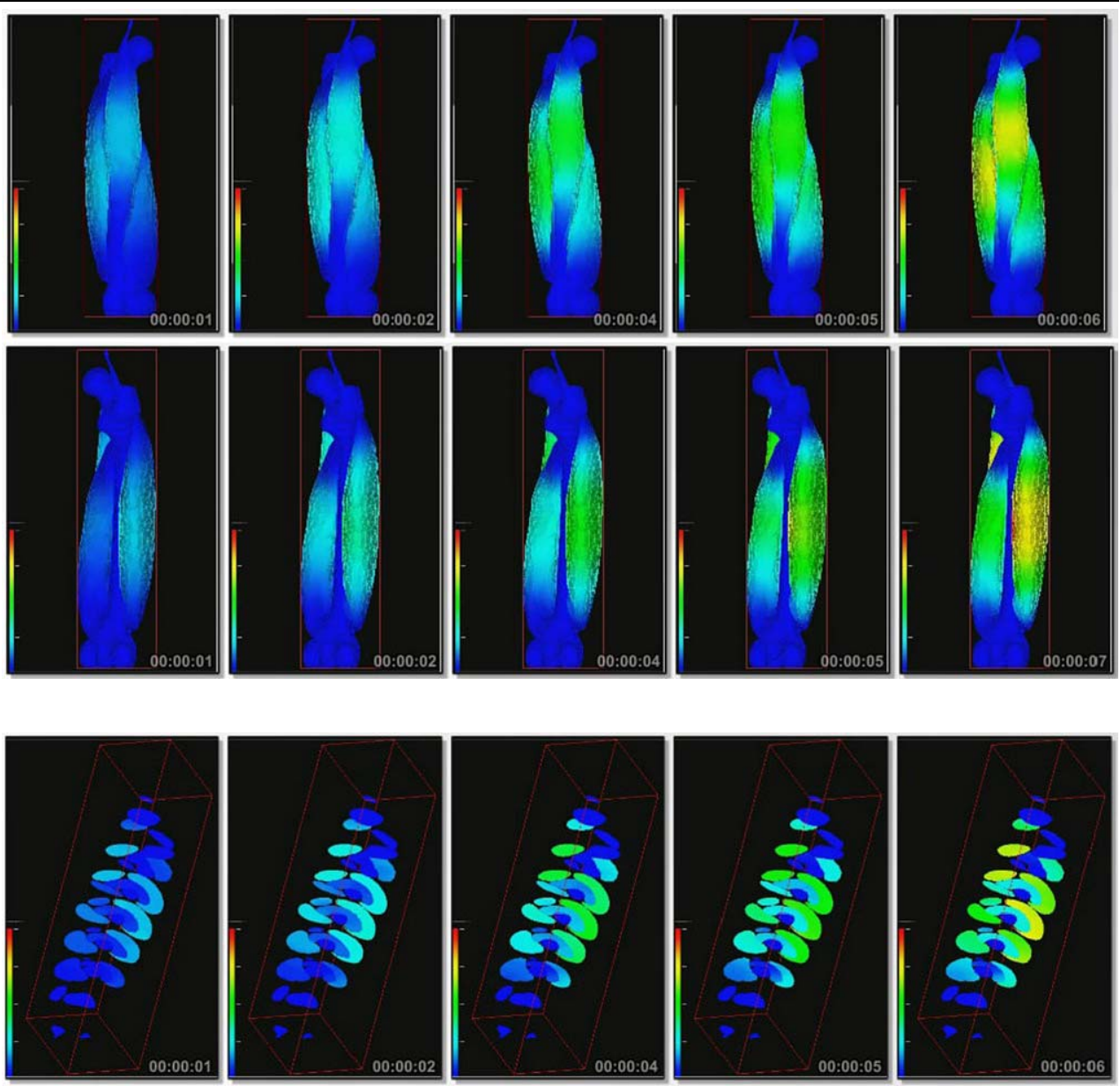

Fig. 7 Internal movements inside the muscles, from 10 slices at 5 regular time-frames of the contraction
The pipeline is almost automatic, and with a few computational optimizations can allow interactive applications. Our model lies on the Finite Element Method which enables an accurate representation of the structure of the fibers.

Two main limitations prevent the production of more precise results. First, the nodal force estimation depends basically on few action lines and global muscular forces. To be more realistic, we should use more accurate data, such as the real directions of the fibers computed from Diffusion Tensor Imaging or Ultrasound capture systems. The distribution of the muscular force on the elements could also be improved, for example using Dynamic MRI data to drive the discretization of the muscles. Second, the contention model produces too restrictive constraints. The resulting deformations appear smal ler than expected. The best way to solve this problem seems to use 3D models with higher resolution, especially near to the contact surfaces between muscles. Indeed, we then could reduce the relative number of constraints to finally free more deformations. But of course, the solving time will increase and may lead to prohibitive calculations. Others reasons for these low nodal displacements could be found such as under estimations of the muscle forces during the inverse dynamics analysis or over-constrained boundary conditions (from too large tendon extremities areas for example).

To conclude, we believe that this solution can help to understand the functionality of a muscle of a specific subject. The estimation of the deformation of a muscle during effort is an important issue in medical applications and diagnostics. To create a platform for the modeling and the functional simulation of subject-specific data is very challenging and we proposed in this paper a new step for its achievement.

Acknowledgements This work is supported by the European Marie Curie Program under the 3D ANATOMICAL HUMAN project (MRTNCT-2006-035763).

\section{References}

1. Code-Aster Platform: http://www.code-aster.org/

2. Salome Platform: http://www.salome-platform.org/

3. Ansari, M., Lee, S., Cho, C.: Hyperelastic muscle simulation. Key Eng. Mater. 345-346, 1241-1244 (2007)

4. Beillas, P., Begeman, P., Yang, K., King, A., Arnoux, P., Kang, H., Kayvantash, K., Brunet, C., Cavallero, C., Prasad, P.: Lower limb: advanced FE model and new experimental data. Stapp Car Crash J. 45, 469-494 (2001) 
5. Belytschko, T., Chen, J.: Meshfree and Particle Methods. Wiley, New York (2007)

6. Belytschko, T., Liu, W., Moran, B.: Nonlinear Finite Elements for Continua and Structures. Wiley, New York (2006)

7. Blankevoort, L., Kuiper, J., Huiskes, R., Grootenboer, H.: Articular contact in a three-dimensional model of the knee. J. Biomech. 24(11), 1019-1031 (1991)

8. Buchler, P., Ramaniraka, N., Rakotomanana, L., Iannotti, J., Farron, A.: A finite element model of the shoulder: application to the comparison of normal and osteoarthritic joints. Clin. Biomech. 17, 630-639 (2002)

9. Crisfield, M.: Non-Linear Finite Element Analysis of Solids and Structures, vols. 1-2 (1997)

10. Delp, S., Loan, J., Hoy, M., Zajac, F., Topp, E., Rosen, J.: An interactive graphics-based model of the lower extremity to study orthopaedic surgical procedures. IEEE Trans. Biomed. Eng. 37(8), 757-767 (1990)

11. Donzelli, P., Spilker, R., Ateshian, G., Mow, V.: Contact analysis of biphasic transversely isotropic cartilage layers and correlations with tissue failure. J. Biomech. 32(10), 1037-1047 (1999)

12. Hirokawa, S., Tsuruno, R.: Hyper-elastic model analysis of anterior cruciate ligament. Med. Eng. Phys. 19(7), 637-651 (1997)

13. James, D., Pai, D.: Dyrt: Dynamic response textures for real time deformation simulation with graphics hardware. ACM Trans. Graph. (SIGGRAPH Proc.) 21 (2002)

14. Jonkers, I., Spaepen, A., Papaioannou, G., Steward, C.: Contribution of forward simulation techniques to the understanding of muscle activation patterns in mid-stance phase of gait. J. Biomech. 35(5), 609-619 (2002)

15. Keyak, J., Skinner, H.: Three-dimensional finite element modelling of bone: effects of element size. J. Biomed. Eng. 14(6), 483-489 (1992)

16. Mac Donald, B.: Practical Stress Analysis with Finite Elements. Glasnevin Publishing (2007)

17. Macosko, C.: Rheology: Principles, Measurements, and Applications. VCH, New York (1994)

18. Mow, V., Flatow, E., Ateshian, G.: Biomechanics. In: Buckwalter, J.A., Einhorn, T.A., Simon, S.R. (eds.) Orthopaedic Basic Science: Biology and Biomechanics of the Musculoskeletal System, 2nd edn. pp. 133-181. American Academy of Orthopaedic Surgeons, USA (2000)

19. Muller, M., Dorsey, J., McMillan, L., Jagnow, R., Cutler, B.: Stable real-time deformations. In: Proceedings of the ACM SIGGRAPH Symposium on Computer Animation, pp. 49-54. ACM, New York (2002)

20. Muller, M., McMillan, L., Dorsey, J., Jagnow, R.: Real-time simulation of deformation and fracture of stiff materials. In: Computer Animation and Simulation '01, Eurographics Workshop, pp. 99111. Eurographics Assoc. (2001)

21. Papaioannou, G., Nianios, G., Mitrogiannis, C., Fyhrie, D., Tashman, S., Yang, K.: Patient-specific knee joint finite element model validation with high-accuracy kinematics from biplane dynamic roentgen stereogrammetric analysis. J. Biomech. 41(12), 26332638 (2008)

22. Scheepers, F., Parent, R., Carlson, W., May, S.: Anatomy-based modeling of the human musculature. In: SIGGRAPH '97: Proceedings of the 24th Annual Conference on Computer Graphics and Interactive Techniques, pp. 163-172 (1997)

23. Schmid, J., Sandholm, A., Chung, F., Thalmann, D., Delingette, H., Magnenat-Thalmann, N.: Musculoskeletal simulation model generation from MRI data sets and motion capture data. The Visual Computer (2009)

24. Sederberg, T., Parry, S.: Free-form deformations of solid geometric models. Comput. Graph. (SIGGRAPH Proc.) pp. 151-160 (1986)
25. Tang, C., Tsui, C., Stojanovic, B., Kojic, M.: Finite element modelling of skeletal muscles coupled with fatigue. Int. J. Mech. Sci. 49(10), 1179-1191 (2007)

26. Thalmann, D., Jianhua, S., Chauvineau, E.: Fast realistic human body deformations for animation and VR applications. In: Proceedings. Computer Graphics International. IEEE Comput. Soc. Press (1996)

27. Winters, J.: Hill-based muscle models: A systems engineering perspective. In: Winters, J.M., Woo, S.L.-Y. (eds.) Multiple Muscle Systems: Biomechanics and Movement Organization, pp. 69-93. Springer, Berlin (1990)

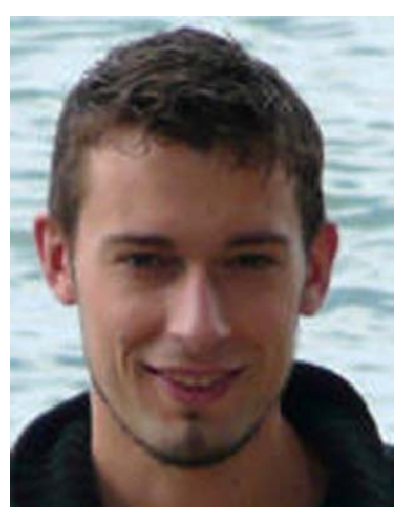

Xavier Maurice received the B.Sc. degree in Computer Science in 2005 from the Robert Schuman Technological University, Strasbourg, France. In 2008, he held Master's degree position at the engineering high school "Institut National des Sciences Appliquées (INSA) de Lyon, France," specializing in Computer Science. Also, he was engaged as an engineer within an international program exchange at EPFL, Switzerland. His research interests are vision, image processing, computer graphics, augmented/virtual reality and $3 \mathrm{D}$ modeling.

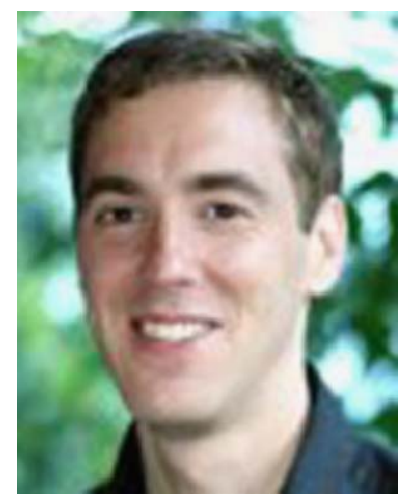

Anders Sandholm is a Ph.D. candidate and a Marie Currie Early Stage Researcher supervised by Professor Daniel Thalmann, Vrlab, at Ecole Polytechnique Fédérale de Lausanne, Switzerland. After having received his M.Sc., aimed toward media technology and visualization, Anders worked as an IT consultant in Stockholm, before joining the eHealth Institute in Kalmar and The Programming Environments Laboratory, Linkopings University. During this year, Anders' main research topic was in evaluation of whether and how an object-oriented language could be used in medical simulators that use haptic devices or accurate tracking devices.

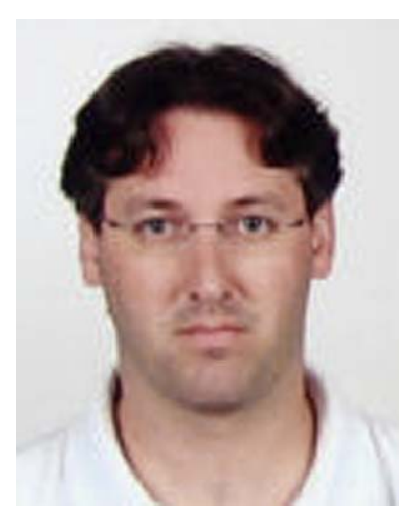

Nicolas Pronost is a postdoctoral researcher in the VRLab at EPFL, Switzerland. He studied Computer Science at the University of Rennes 1 (France), where he Received his M.Sc. degree in 2003. He then worked within the SIAMES Project of the INRIA/IRISA Laboratory (Rennes, France) where he received his Ph.D. in Computer Science from the University of Rennes 1 in December 2006, and then he was a postdoctoral researcher at the State Key Lab of CAD \& CG at Zheijiang University (China). The main topics of his work are the analysis and the synthesis of human motions using normalized approaches and musculoskeletal simulations. 


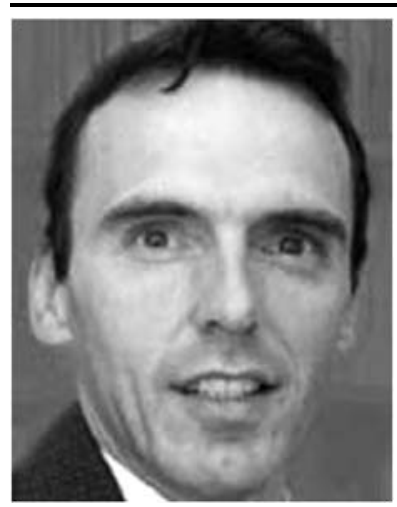

Ronan Boulic is a Senior Researcher, Lecturer and Ph.D. Director at EPFL. $\mathrm{He}$ is working in the Virtual Reality $\mathrm{Lab}$ and his research interests include realistic motion synthesis for virtual humans and robots. He received the Ph.D. degree in Computer Science in 1986 from the University of Rennes, France, at the INRIA-IRISA research institute. $\mathrm{He}$ received the Habilitation degree from the University of Grenoble, France, in March 1995.

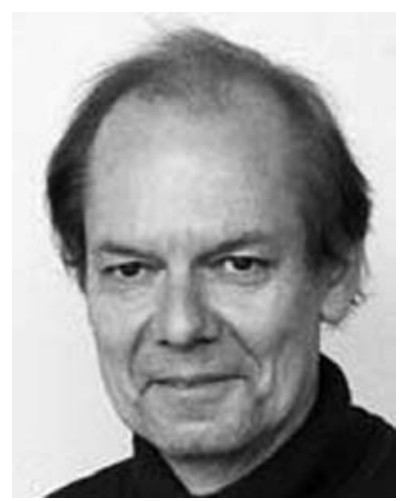

Daniel Thalmann is Professor and Director of The Virtual Reality Lab (VRlab) at EPFL, Switzerland. He is a pioneer in research on virtual humans. His current research interests include real-time virtual humans in virtual reality, networked virtual environments, artificial life, and multimedia. Daniel Thalmann has been Professor at the University of Montreal and Visiting Professor/Researcher at CERN, University of Nebraska, University of Tokyo, and Institute of System Science in Singapore. He received his Ph.D. in Computer Science in 1977 from the University of Geneva and an Honorary Doctorate (Honoris Causa) from University Paul-Sabatier in Toulouse, France, in 2003. 